\title{
HIV-related stigma and discrimination amongst healthcare providers in Guangzhou, China
}

\author{
Xiaomei Dong ${ }^{1}$, Jianwei Yang ${ }^{1}$, Lin Peng ${ }^{2}$, Minhui Pang ${ }^{1}$, Jiayi Zhang ${ }^{1}$, Zhan Zhang ${ }^{3}$, Jiaming Rao ${ }^{1}$,
} Haiqing Wang ${ }^{1}$ and Xiongfei Chen ${ }^{4^{*}}$

\begin{abstract}
Background: HIV-related discrimination amongst healthcare providers is one of the strongest obstacles to effectively responding to HIV. This study was conducted to explore the occurrence of and other factors related to discrimination against people living with HIV/AIDS amongst healthcare providers in Guangzhou, China.

Methods: This was a cross-sectional study, conducted between July and October 2016, that enrolled healthcare providers from 9 healthcare institutions in Guangzhou, China. HIV-related discrimination was assessed using anonymous self-designed questionnaires. Chi-square tests were used to study the differences in the socio-demographic characteristics, occupational characteristics, HIV-related knowledge and personal attitudes between participants who had and had not discriminated against People living with HIV/AIDS (PLWHA). A multivariate logistic regression analysis was used to study the factors associated with HIV-related discrimination.

Results: A total of 972 healthcare providers were investigated, and 386 (39.7\%) had previously served HIVpositive individuals in their work. Administering HIV antibody tests for patients without his or her consent was the most frequent act of discrimination (65.3\%), and other forms of discrimination, including "differential treatment" (51.0\%), "disclosed information" (46.4\%) and "refused to treat" (38.6\%), were also prevalent. The logistic regression analysis indicated that people who had worked for 3-7 years, worked in secondary hospitals or lower, worked in surgical departments, had lower scores on HIV transmission knowledge, were dissatisfied with the occupational exposure protection system offered by the government, were worried about HIV-related exposure and feared HIV-related exposure were more likely to commit an act of medical discrimination against PLWHA.

Conclusion: HIV-related discrimination was not unusual in the healthcare providers of Guangzhou, which may be related to their negative cognitions and attitudes as well as the hospital management system and government policy. Therefore, comprehensive HIV-related knowledge education should be implemented to change the attitude of healthcare providers. In addition, the current laws and regulations should be refined by the government to protect the rights of healthcare providers. The contradiction between designated hospitals and non-designated hospitals should be resolved to ensure that PLWHA receive timely and effective help and treatment.
\end{abstract}

Keywords: HIV/AIDS, Healthcare providers, Stigma and discrimination

\footnotetext{
* Correspondence: 11742513@qq.com

${ }^{4}$ Guangzhou Centers for Disease Control and Prevention, Guangzhou,

Guangdong, China

Full list of author information is available at the end of the article
}

(c) The Author(s). 2018 Open Access This article is distributed under the terms of the Creative Commons Attribution 4.0 International License (http://creativecommons.org/licenses/by/4.0/), which permits unrestricted use, distribution, and reproduction in any medium, provided you give appropriate credit to the original author(s) and the source, provide a link to the Creative Commons license, and indicate if changes were made. The Creative Commons Public Domain Dedication waiver (http://creativecommons.org/publicdomain/zero/1.0/) applies to the data made available in this article, unless otherwise stated. 


\section{Background}

HIV stigma is prevalent worldwide. The Joint United Nations Program on HIV/AIDS (UNAIDS) defines HIV-related stigma and discrimination as follows: "a process of devaluation of people either living with or associated with HIV and AIDS" [1, 2]. HIV/AIDS-related stigma and discrimination occur in many settings, but they may have more serious consequences in healthcare settings [3-5]. In recent years, many media outlets have frequently portrayed HIV-positive patients encountering several difficulties in the process of seeking medical treatment in China, particularly the case of Xiao Feng, an AIDS patient. To receive a surgical procedure that two hospitals denied him, he eventually received the surgery he needed after concealing his HIV-positive status, which propelled public discussion [6-8].

"HIV is dreadful, but discrimination is even more dreadful," said Cai Weiping, a director of the infectious diseases department at Guangzhou No. 8 People's Hospital. Stigmatizing attitudes and behaviours towards people living with HIV/AIDS (PLWHA) not only interfere with their decisions to seek HIV counselling and testing, the prevention of mother-to-child transmission, and the likelihood of disclosing their HIV status [3, 9], but these attitudes and behaviours can also hinder the progress of AIDS prevention and control. In addition, discrimination from the outside world will lead to negative emotions amongst PLWHA, including anxiety, depression, guilt, and other mental health symptoms [10, 11].

In China, to ensure that AIDS patients have access to medical care, employment and privacy, the government has developed a number of laws and regulations, such as the Law of the People's Republic of China on the Prevention and Treatment of Infection Diseases (2004), the Regulations on AIDS Prevention and Treatment (2006) [12], and the Tort Law of the People's Republic of China (2009) [13]. However, the government's guide on how hospitals should receive HIV/AIDS patients is not sufficiently clear, and some medical facilities use the designated hospitals as an excuse to reject patients from receiving routine medical services. The designated hospitals are selected by the Ministry of Health to treat PLWHA, particularly those requiring antiviral treatment. However, most designated hospitals are specialized hospitals that only address infectious diseases and are not qualified to perform major surgeries. This phenomenon may lead to concealed illnesses of patients and cause irreparable damage to society. Therefore, the serious problem of HIV/AIDS discrimination commands attention $[14,15]$.

HIV stigma and discrimination have been extensively documented amongst healthcare workers (HCWs) worldwide [5, 16-19]. To combat stigma and discrimination, it is important to explore their associated factors and to explore how they vary across cultural contexts within a country [20]. Nevertheless, in the Chinese context, HIV-related discrimination in medical institutions was conducted mainly amongst PLWHA, and fewer studies have investigated the phenomena from the perspective of HCWs. Moreover, most studies focus on discriminatory attitudes, with occasional studies involving specific discriminatory behaviours. In this study, we selected medical staff members as participants, investigated their attitudes and behaviours towards HIV-positive patients, and explored the influencing factors of discriminatory behaviours, which will provide countermeasures and references for relevant departments to reduce discrimination in the medical field, protect the medical interests of PLWHA and promote HIV prevention and control.

\section{Methods}

\section{Study population, setting and design}

This study is a cross-sectional survey of a convenience sample of 972 health care providers from different grades of healthcare facilities in Guangzhou. The study was conducted in 2016 with the goal of assessing HIV-related discrimination and behavioural risk factors in healthcare providers. Guangzhou is located in the Pearl River Delta area and has a high HIV infection rate in China. To meet the inclusion criteria, the participants were required to have worked as a doctor, nurse or laboratory staff member in a current healthcare setting for at least one year and be able to answer questions without assistance. All participants provided written informed consent. All procedures were approved by the Ethical Committee of the Guangzhou Centers for Disease Control and Prevention.

\section{Sampling procedure}

The sample content was calculated according to the following formula: $n=z^{2} p(1-p) / d^{2}$. Our study established a $95 \%$ confidence level $(z=1.96)$; tolerance was defined as 0.03 . According to the pre-investigation results, the incidence of medical discrimination was $70 \%(P=0.7$, $1-P=0.3)$. To reduce the problem of an insufficient sample size caused by missing or incomplete data from the questionnaire, the sample size was increased by $20 \%$ from the original. In total, the sample size was 1075 . Considering the classified management system of medical organizations issued by the Ministry of Health of the People's Republic of China in 1989, public hospitals are classified into three grades (tertiary hospitals are better than secondary hospitals, which are in turn better than primary hospitals). To improve the sample representation, a stratified sampling method was adopted. We selected 4 tertiary hospitals, 3 secondary hospitals and 2 primary hospitals. Taking into account the personnel 
size and department settings in the different levels of hospitals, 150-200 questionnaires were distributed in the tertiary hospitals, 80-100 questionnaires were distributed in the secondary hospitals, and 30-50 questionnaires were distributed in the primary hospitals. A total of 1075 questionnaires were distributed; 1031 questionnaires were withdrawn, and 972 completed questionnaires were obtained, yielding response and completion rates of 95.91 and $94.28 \%$, respectively.

\section{Data collection procedure}

The data concerning the HCWs' attitudes and acts towards PLWHA were collected using a self-administered questionnaire, which was created on the basis of literature references, pilot tests and expert optimization [3-5, 21]. The investigation was conducted with the help of the Department of Hospital Infection Management in each hospital. The questionnaires were completed by the medical staff during leisure time at work. Before the formal investigation, the $972 \mathrm{HCWs}$ who were eligible for enrolment were asked to sign the informed consent form. The questionnaire took an average of $15 \mathrm{~min}$ to complete. After the HCWs independently finished their own questionnaire, we reviewed the questionnaire and then arranged and numbered the questionnaires at that time.

\section{Measures}

The questionnaires comprised 4 parts, including socio-demographic variables, occupational characteristics, HIV-related knowledge, personal attitudes and discrimination behaviours.

\section{HIV-related knowledge}

HIV-related knowledge contained 2 parts, including HIV transmission knowledge and exposure prevention knowledge (items are listed in Table 2).

- The HIV transmission knowledge section included 6 questions. For each item, the responses were coded as 1 (correct answer) or 0 (incorrect answer or unknown). The number of correct responses was summed. A higher score reflected better knowledge. Because the median knowledge score was 4 , the respondents with correct answers to at least 4 questions represented the high-score group, and those with correct answers to less than 4 questions represented the low-score group.

- Exposure prevention knowledge was used to measure the emergency response ability of the medical staff after HIV exposure at work. This module contained 10 questions. Respondents with correct responses to at least six of the ten exposure prevention knowledge questions were defined as the high-score group, and those with less than 6 correct responses were defined as the low-score group.

\section{Personal attitudes}

The personal attitudes section contained 3 parts, including willingness to treat, satisfaction with the security system and fear of HIV exposure.

- Willingness to treat: Two individual items measured the medical workers' willingness to take care of PLWHA: (i) general treatment and (ii) high-risk treatment (yes or no).

- Evaluation of occupational exposure protection system: The evaluation of the current security system offered by the government for HIV occupational exposure had one question measured on a three-point scale $(1=$ not at all to $3=$ very satisfied).

- Fear of HIV exposure: The fear of work-related HIV exposure had one question measured on a threepoint scale $(1=$ no fear, $2=$ some fear, and $3=$ substantial fear).

\section{Discrimination behaviours}

This module was completed by participants who had experience in the diagnosis and treatment of PLWHA. Guided by the Protocol for the Identification of Discrimination against People Living with HIV (UNAIDS, 2000), the following four questions about HIV-related discrimination in health care were included: (1) Did you ever refuse to treat patients on the grounds of HIV/AIDS status (refused to treat)? (2) Did you ever administer an HIV antibody test for patients without his or her consent (forced detection)? (3) Did you ever administer a different treatment for patients on the grounds of HIV/AIDS status (differential treatment)? (4) Did you ever disclose a patient's HIV status to a colleague who was not directly involved in the management of that case (disclosed information)? The response options for each statement were yes or no.

\section{Statistical analysis}

The data were checked for integrity and were entered into Epi-data 3.1. The data analysis was performed using SPSS 19.0. Descriptive statistics were used to describe the health care providers' demographic characteristics and occupational characteristics. In addition, we explored differences in the socio-demographic characteristics, occupational characteristics, HIV-related knowledge and personal attitudes between participants who had and had not discriminated against PLWHA by using chi-square tests $(\alpha=0.05)$. Furthermore, a multivariate logistic regression analysis was used to study the related 
factors of four types of HIV-related discrimination, and the factors were screened by the likelihood ratio approach method $(\alpha=0.05)$.

\section{Results}

\section{Sociodemographic and clinical characteristics}

As shown in Table 1, 972 healthcare workers were included in the sample. There were 706 females, representing $72.6 \%$ of the sample. Approximately $44.2 \%$ of the respondents had been working at their current health care setting for less than 2 years, $31.6 \%$ had been working there for more than 8 years, $66.4 \%$ of the sample came from tertiary hospitals, and $45.0 \%$ of the participants were doctors. Amongst all participants, 53.4\% reported having received training on occupational

Table 1 Sociodemographic characteristics of healthcare providers, Guangzhou, China

\begin{tabular}{lll}
\hline Variable & Number & Percent \\
\hline Gender & & \\
Male & 266 & 27.4 \\
Female & 706 & 72.6
\end{tabular}

\begin{tabular}{lll}
\multicolumn{3}{c}{ Working years } \\
$\leq 2$ years & 430 & 44.2 \\
$3-7$ years & 235 & 24.2 \\
$\geq 8$ years & 307 & 31.6
\end{tabular}

Medical education

$\begin{array}{lll}\text { Junior college degree or lower } & 396 & 40.7 \\ \text { Medical degree } & 428 & 44.0 \\ \text { Higher than medical degree } & 148 & 15.2\end{array}$

Level of care

Tertiary hospital 645

\begin{tabular}{|c|c|c|}
\hline \multicolumn{3}{|c|}{ Training } \\
\hline Yes & 519 & 53.4 \\
\hline No & 453 & 46.6 \\
\hline \multicolumn{3}{|c|}{ Profession } \\
\hline Doctor & 437 & 45.0 \\
\hline Nurse & 432 & 44.4 \\
\hline Laboratory technician & 103 & 10.6 \\
\hline
\end{tabular}

\begin{tabular}{lll}
\multicolumn{1}{c}{ Department } & & \\
Internal medicine & 266 & 27.4 \\
Surgical department & 355 & 36.5 \\
Clinical laboratory department & 66 & 6.8 \\
Administrative department & 285 & 29.3 \\
\hline
\end{tabular}

exposure protection skills, and 59.2\% had received an undergraduate education or above.

\section{HIV-related knowledge}

The aggregate knowledge score was 10.74 (67.13 out of 100). The mean HIV transmission knowledge score was 4.09. Knowledge regarding the route of exposure was high, with $84.2 \%$ of respondents answering accurately that the disease could not be spread by intact skin coming in contact with HIV-infected blood. Knowledge was somewhat deficient regarding the AIDS epidemic in China. The results for HIV transmission knowledge by item are displayed in Table 2. The mean score of HIV exposure knowledge was 6.65. The item with the highest rate of correct answers was "How should one address needles used by HIV-positive patients?" The item with the lowest rate of correct answers was "Which is the correct operation to prevent occupational exposure to HIV?" The results for exposure prevention knowledge by item are displayed in Table 2.

\section{Personal attitudes at work}

The participants' attitudes towards PLWHA are presented in Table 3. In total, $33.0 \%$ of healthcare workers reported that they were satisfied with the protection system offered by the government for HIV occupational exposure, whereas $57.2 \%$ were neutral regarding the system. Approximately $80 \%$ of participants indicated that they were worried about work-related HIV transmission. When asked about the willingness to treat HIV-positive patients, $85.2 \%$ of participants responded that they would provide general treatment for PLWHA, whereas $78.7 \%$ would provide high-risk treatment for PLWHA.

\section{Discrimination against PLWHA}

Amongst the 972 respondents, 386 (39.7\%) reported they had experience in the diagnosis and treatment of PLWHA, whereas 586 (60.3\%) had no such experience. We found that $300(77.72 \%)$ HCWs had discriminated against PLWHA in the process of providing medical attention. Administering HIV antibody tests for patients without his or her consent was the act of discrimination reported by the greatest proportion of healthcare providers $(65.3 \%)$. The act of discrimination that was reported by the second-highest proportion of healthcare providers (51.0\%) was providing differential treatment to patients based on HIV status. Furthermore, $46.4 \%$ of healthcare providers indicated that they had disclosed a patient's HIV status to a colleague who had not been directly involved in the management of the case, and $38.6 \%$ reported that they had refused to treat PLWHA in the past (Table 4). 
Table 2 HIV-related knowledge of healthcare providers, Guangzhou, China

\begin{tabular}{|c|c|c|}
\hline & Item & Correct responses $\mathrm{N}(\%)$ \\
\hline \multirow{6}{*}{$\begin{array}{l}\text { HIV transmission } \\
\text { knowledge }\end{array}$} & Which is the least risky pattern of exposure to HIV-positive blood? & $818(84.2)$ \\
\hline & Which type of body fluid from PLWHA can cause infection? & $797(82.0)$ \\
\hline & Which is the most effective measure to prevent HIV infection? & $769(79.1)$ \\
\hline & What is the current stage of the AIDS epidemic in China? & $752(77.4)$ \\
\hline & Which sexual behaviour is the most dangerous? & $470(48.4)$ \\
\hline & What are the epidemic characteristics of HIV/AIDS in China? & $379(39.0)$ \\
\hline \multirow{10}{*}{$\begin{array}{l}\text { Exposure prevention } \\
\text { knowledge }\end{array}$} & How should one address needles used by HIV-positive patients? & $879(90.4)$ \\
\hline & $\begin{array}{l}\text { Which is not a universal precautionary principle to prevent occupational } \\
\text { exposure to HIV? }\end{array}$ & $807(83.0)$ \\
\hline & Which is not a measure of HIV occupational exposure? & $741(76.2)$ \\
\hline & What is the best time for preventive medication after occupational exposure? & $720(74.1)$ \\
\hline & Which treatment is not correct if stabbed by HIV-contaminated needles? & $681(70.1)$ \\
\hline & $\begin{array}{l}\text { Which is not a principle that is commonly followed by parties and the relevant } \\
\text { departments after HIV occupational exposure? }\end{array}$ & $564(58.0)$ \\
\hline & Which is not the main basis for assessing the risk of HIV exposure? & $556(57.2)$ \\
\hline & How many days is the course of preventive medication after occupational exposure? & $491(50.5)$ \\
\hline & How should one to treat the eye mucosa after it is splashed by HIV-infected fluid? & $460(47.3)$ \\
\hline & Which is the correct operation to prevent occupational exposure to HIV? & $214(22.0)$ \\
\hline
\end{tabular}

\section{Bivariate results}

Table 5 shows the results of the bivariate associations between discrimination acts and factors. Regarding clinic-level factors, the healthcare providers who worked in tertiary hospitals refused to treat PLWHA and conducted HIV tests without the patients' consent less frequently than those who worked in secondary hospitals or lower $(P<0.05)$. Work seniority differences were

Table 3 Healthcare providers' personal attitudes at work

\begin{tabular}{lc}
\hline Personal attitudes & $N(\%)$ \\
\hline Evaluation of occupational exposure protection system & $321(33.0 \%)$ \\
Satisfaction & $557(57.2 \%)$ \\
Neutral & $94(9.7 \%)$ \\
Dissatisfaction & Fear of work-related HIV exposure \\
& $158(16.3 \%)$ \\
Substantial fear $\quad 611(62.9 \%)$ \\
Some fear $\quad 203(20.9 \%)$ \\
No fear $\quad$ I would provide general treatment for PLWHA \\
\\
Yes & $828(85.2 \%)$ \\
No & $144(14.8 \%)$ \\
& \\
Yes & I would provide high-risk treatment for PLWHA \\
No & $765(78.7 \%)$ \\
\hline
\end{tabular}

found only in the "refused to treat" category, with the workers with 3-7 years of experience being more likely to refuse to treat PLWHA $(P<0.05)$. Healthcare providers who worked in surgical departments reported higher rates of "forced detection" $(P<0.01)$ and "differential treatment" $(P<0.01)$ than those of participants in internal medicine departments.

Concerning the level of HIV-related knowledge, the participants who had higher scores on HIV transmission knowledge reported higher rates of "refused treatment" $(P<0.05)$, "forced detection" and "disclosed information" $(P<0.05)$. In addition, those who reported higher scores on exposure prevention knowledge had higher rates of "refused treatment" $(P<0.05)$. With regard to personal attitudes, the people who were more satisfied with the current occupational exposure protection system had a smaller possibility of having conducted HIV tests without consent $(P<0.01)$. In addition, fearing work-related HIV exposure was a significant predictor of "refused treatment" $(P<0.01)$, "forced detection" $(P<0.01)$ and "differential treatment" $(P<0.01)$.

\section{Multivariate results}

The multivariate regression results are displayed in Table 6. Four models included variables that were significant in their univariate analysis. In the multivariate model of "refused treatment", all factors that were significant in the univariate analysis, in addition to exposure prevention knowledge and training, remained significant influential factors of discrimination. Working 
Table 4 Acts of discrimination by healthcare providers, Guangzhou, China

\begin{tabular}{lllll}
\hline & \multicolumn{1}{l}{ Had experience in the diagnosis and treatment of PLWHA } & Total & Had no \\
\cline { 2 - 4 } & Yes & No & 386 & 586 \\
Refused to treat & $149(38.6)$ & $237(61.4)$ & 386 & 586 \\
Forced detection & $252(65.3)$ & $134(34.7)$ & 386 & 586 \\
Differential treatment & $197(51.0)$ & $189(49.0)$ & 386 & 586 \\
Disclosed information & $179(46.4)$ & $207(53.6)$ & 386 \\
\hline
\end{tabular}

in secondary hospitals or lower, working for 3-7 years, having lower scores on HIV transmission knowledge and fearing HIV-related exposure continued to be significant predictors of higher levels of "refused treatment". The model of "forced detection" showed that working in surgical departments, working in secondary hospitals or lower, being dissatisfied with the occupational exposure protection system offered by the government and being worried about HIV-related exposure were significant factors of higher rates of "forced detection". The factors of department and fear of HIV-related exposure were entered in the model of "differential treatment", and HIV transmission knowledge was entered in the model of "disclosed information".

\section{Discussion}

In the medical field, discrimination and stigma have engendered serious and even tragic consequences, denying the people living with HIV/AIDS access to care and testing, which can in turn increase the likelihood of patients concealing their HIV status [8, 22]. In addition, the chronic nature of HIV necessitates ongoing medical care across a lifespan, increasing the chances of a doctor being exposed to the disease; however, the patient will not receive proper treatment if doctors do not know his or her infection status [23]. In addition, stigma and discrimination have been extensively reported in medical settings worldwide, most of which involved discriminatory attitudes amongst medical workers, with a few cases of discriminatory behaviour. Therefore, it is critical to study the relationship between the attitudes and behaviours of individuals who engage in HIV-related discrimination and to explore the causes of this discrimination.

In our study, we found that $77.7 \%$ of HCWs had discriminated against PLWHA in the process of providing medical attention. One of the most commonly reported forms of discrimination was forced detection, followed by differential treatment, disclosed information and refused treatment. All rates were higher than those reported in a Belizean study by Andrewin A [24]. The reason for these higher rates may be related to the contradiction between designated hospitals and non-designated hospitals that was mentioned previously because doctors always transfer PLWHA to designated hospitals. Additionally, it was noted that healthcare providers give differential treatment and disclose HIV status to facilitate the healthcare given to the clients and to take protective measures when dealing with PLWHA $[5,25]$. However, these acts could be considered discrimination and privacy violations by PLWHA, resulting in irreparable psychological damage to those patients. Therefore, we must determine how to reduce unintentional discrimination in future studies.

Occupational characteristics were found to be significantly associated with acts of HIV-related discrimination in this population. Participants in secondary hospitals or lower had a higher frequency of "refused treatment". In China, compared to tertiary hospitals, which have more advanced equipment and more highly educated staff members, primary hospitals are limited by materials and supplies, and they cannot provide simple treatments, such as haemorrhoid surgery [26]. Similarly, the healthcare providers in surgical departments had a higher frequency of "forced detection" and "differential treatment". The Twelfth Five-Year Action Plan for HIV/ AIDS Prevention and Control in China pointed out that routine examination for inpatients and outpatients will include HIV testing in highly endemic HIV areas. Because it is routine testing before surgery, medical staff members often forget to inform the patient, thus violating the HIV-positive patient's rights. Therefore, we strongly recommend that providers increase interactions with PLWHA before treatment.

Negative attitudes of health professionals, which are key factors in dealing with PLWHA, may result in undesirable consequences [27]. In the present study, the people who were more satisfied with the occupational exposure protection system had a lower frequency of "forced detection". One study amongst healthcare providers in Southwest Ethiopia, for example, found that the perception of protocol-related institutional support could reduce the stigma score ${ }^{5}$. Another study by LiLi detected similar trends [28]. These findings highlight the importance of establishing institutional support in the work of eliminating discrimination. However, only one-third of respondents in this survey were satisfied with the occupational exposure protection system. Thus, most hospitals lack a strict series of AIDS occupational 


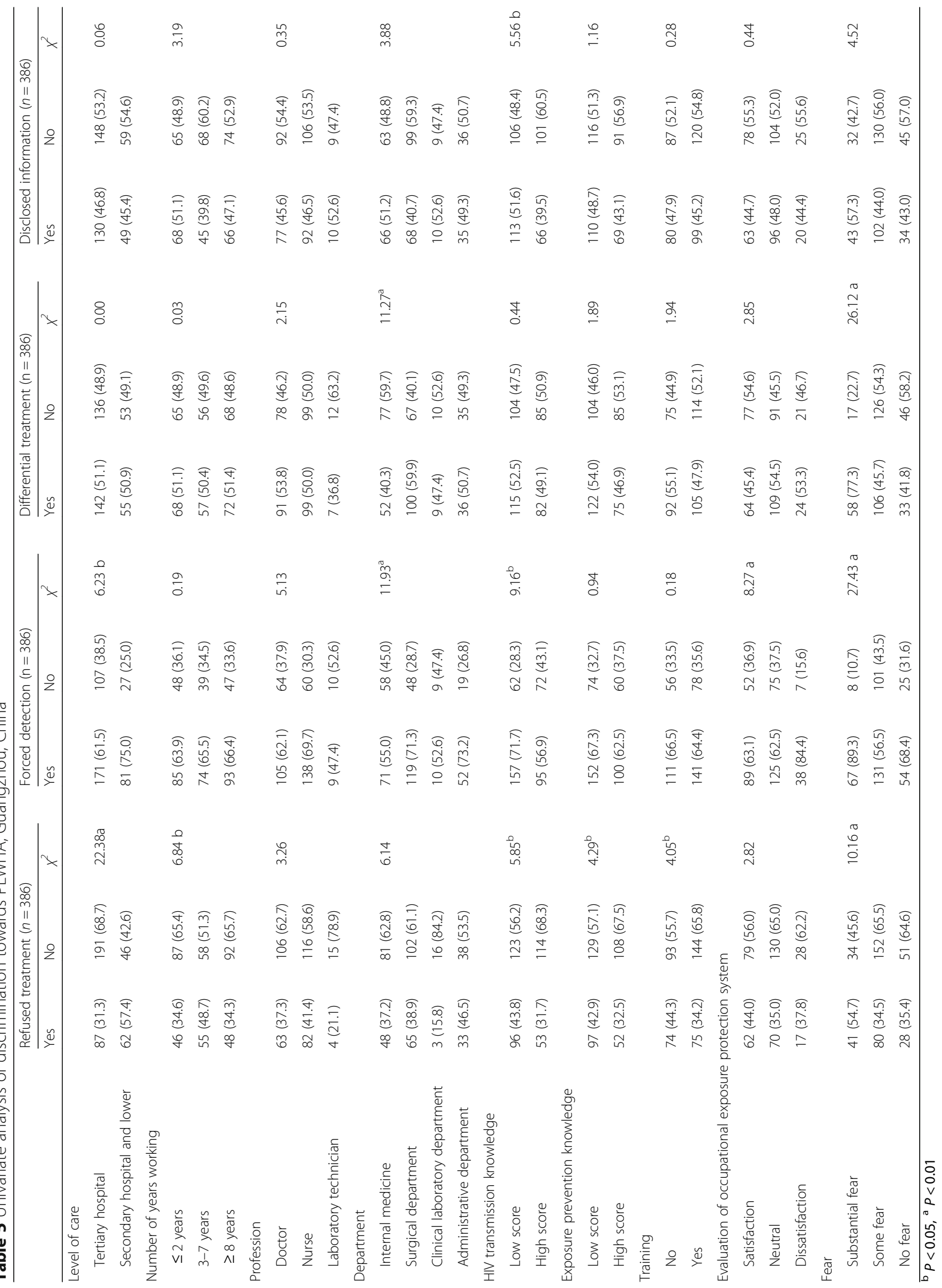


Table 6 Multivariate regression analysis of discrimination towards PLWHA, Guangzhou

\begin{tabular}{|c|c|c|c|}
\hline Discrimination & Variables & Wald & OR $(95 \% \mathrm{Cl})$ \\
\hline \multirow[t]{10}{*}{ Refused treatment $(0=\mathrm{No}, 1=\mathrm{Yes})$} & Level of care (ref: Tertiary hospital) & & \\
\hline & Secondary hospital or lower & 19.405 & $2.997(1.839,4.885)$ \\
\hline & Number of years working (ref: $\geq 8$ years) & 6.097 & \\
\hline & $3-7$ years & 5.179 & $1.877(1.091,3.227)$ \\
\hline & $\leq 2$ years & 0.080 & $1.083(0.624,1.881)$ \\
\hline & HIV transmission knowledge (ref: High score) & & \\
\hline & Low score & 5.797 & $1.741(1.109,2.735)$ \\
\hline & Fear (ref: No fear) & 9.316 & \\
\hline & Some fear & 0.369 & $0.840(0.478,1.474)$ \\
\hline & Substantial fear & 4.115 & $2.021(1.024,3.987)$ \\
\hline \multirow[t]{12}{*}{ Forced detection $(0=\mathrm{No}, 1=\mathrm{Yes})$} & Level of care (ref: Tertiary hospital) & & \\
\hline & Secondary hospital or lower & 5.444 & $1.900(1.108,3.258)$ \\
\hline & Department (ref: Internal medicine) & 12.558 & \\
\hline & Surgical department & 9.202 & $2.221(1.326,3.720)$ \\
\hline & Clinical laboratory department & 0.411 & $0.710(0.249,2.024)$ \\
\hline & Administrative department & 4.249 & $2.031(1.035,3.985)$ \\
\hline & $\begin{array}{l}\text { Evaluation of Occupational Exposure } \\
\text { Protection System (ref: Satisfaction) }\end{array}$ & 6.638 & \\
\hline & Neutral & 0.053 & $1.058(0.653,1.714)$ \\
\hline & Dissatisfaction & 6.320 & $3.276(1.299,8.262)$ \\
\hline & Fear (ref: No fear) & 23.348 & \\
\hline & Some fear & 3.440 & $0.585(0.332,1.031)$ \\
\hline & Substantial fear & 9.113 & $4.014(1.628,9.898)$ \\
\hline \multirow[t]{7}{*}{ Differential treatment $(0=\mathrm{No}, 1=$ Yes $)$} & Department (ref: Internal medicine) & 13.779 & \\
\hline & Surgical department & 13.490 & $2.520(1.539,4.127)$ \\
\hline & Clinical laboratory department & 0.393 & $1.383(0.502,3.815)$ \\
\hline & Administrative department & 1.647 & $1.492(0.810,2.751)$ \\
\hline & Fear (ref: No fear) & 25.99 & \\
\hline & Some fear & 0.819 & $1.277(0.752,2.169)$ \\
\hline & Substantial fear & 21.741 & $5.583(2.710,11.504)$ \\
\hline \multirow[t]{2}{*}{ Disclosed information ( $0=$ No, 1 = Yes) } & HIV transmission knowledge (ref: High score) & & \\
\hline & Low score & 5.527 & $1.631(1.085,2.453)$ \\
\hline
\end{tabular}

protection training, protective equipment or rules and regulations, which objectively hinders the supply of medical personnel health services.

In our study, fear of occupational exposure was a key factor in the occurrence of medical discrimination. Other studies have also shown that worry about infection at work favours discriminatory attitudes ${ }^{3}$. Therefore, reducing fear is also an urgent problem to be addressed. To maintain the interests of the medical staff members and eliminate their fears, the National Health and Family Planning Commission of the PRC released the latest version of the "Occupational Diseases Classification and Catalog" with the inclusion of AIDS (limited to health staff and policemen) for the first time in 2013; however, the amount of compensation is not clear, and the process is complicated. Therefore, the claims system must be further improved. In addition, preventive measures should also be strengthened. From the aspects of medical staff members, HIV-related training should be conducted to address unwarranted fears about the risk of infection with HIV after casual contact. From the governmental perspective, the medical investment should be increased to ensure that there are adequate hardware facilities to prevent the infection of healthcare workers.

Similar to other studies, ours has several limitations that must be considered when interpreting the results. The data were collected from only one city; thus, caution should be exercised in generalizing these findings to 
a different population or other geographic locations. However, Guangzhou, as the capital city of Guangdong Provence, has a wide range of services. In addition, we made every effort to recruit healthcare providers from different levels of hospitals. Therefore, our findings provide important support for the need for HIV-related stigma-reduction interventions in the Chinese healthcare setting.

\section{Conclusions}

Our findings revealed that HIV-related discrimination was not unusual in the healthcare providers of Guangzhou, including "Refused to treat", "Forced detection", "Differential treatment", and "Disclosed information", which were related to the health care workers' negative cognitions and attitudes, the hospital management system and government policy. Therefore, future treatment and support for AIDS patients should focus more on changing the HCWs' cognitions and attitudes towards PLWHA to ensure that PLWHA can receive timely and effective help and treatment. The reduction of medical discrimination was inseparable from government policy support and the hospital management system. The government should implement measures to refine the current laws and regulations and establish a professional insurance mechanism to protect the rights of medical staff members, thereby reducing the worries of HCWs in providing services to AIDS patients. The hospital should attend to the contradiction between designated hospitals and non-designated hospitals, increase investment and improve service capacity to address the rejection caused by the lack of technology and equipment.

\section{Abbreviations}

HCW: Healthcare worker; PLWHA: People living with HIV/AIDS; PRC: People's Republic of China; UNAIDS: The Joint United Nations Program on HIV/AIDS

\section{Funding}

This study was supported by the Medical Scientific Research Foundation of Guangdong Province, China (A2013336).

\section{Availability of data and materials}

The datasets generated and/or analysed during the current study are not publicly available because of the confidentiality principle of the research project.

\section{Authors' contributions}

The study was designed by XD, XC, JY and LP. Data collection was performed by $X D, J Y, M P, J Z, Z Z, J R$ and HW. XD, XC, JY, LP, MP, JZ, ZZ, JR and HW critically reviewed the manuscript and the interpretation of the results. All authors read and approved the final manuscript.

\section{Authors' information}

Xiaomei Dong is an Associate Professor at Jinan University, Guangzhou, China (PhD). Xiongfei Chen and Lin Peng are public health doctors (MA). Jianwei Yang, Minhui Pang, Jiayi Zhang, Zhan Zhang, Jiaming Rao and Haiqing Wang are postgraduate students at Jinan University, Guangzhou, China.

\section{Ethics approval and consent to participate}

All procedures were approved by the Ethical Committee of the Guangzhou Centers for Disease Control and Prevention. Prior to the survey, the healthcare providers who participated in the study were introduced to the objectives of the study by the research team and signed the informed consent.

\section{Competing interests}

The authors declare that they have no competing interests.

\section{Publisher's Note}

Springer Nature remains neutral with regard to jurisdictional claims in published maps and institutional affiliations.

\section{Author details}

'Department of Epidemiology, School of Basic Medicine, Jinan University, Guangzhou, China. ${ }^{2}$ Dongguan Municipal Centers for Disease Control and Prevention, Dongguan, Guangdong Province, China. ${ }^{3}$ Department of Medical Statistics, School of Basic Medicine, Jinan University, Guangzhou, China.

${ }^{4}$ Guangzhou Centers for Disease Control and Prevention, Guangzhou, Guangdong, China.

Received: 8 August 2017 Accepted: 1 June 2018

Published online: 15 June 2018

\section{References}

1. Patankar N, PanditD. Study of HIV/AIDS related stigma and discrimination in people living with HIV AIDS. J Res Med Dent Sci 2014; 2(4):53-56.

2. Centers for Disease Control and Prevention. HIV-related knowledge and stigma-United States, 2000. JAMA. 2000;284(24):3118.

3. Ekstrand ML, RamakrishnaJ BS, Heylen E. Prevalence and drivers of HIV stigma among health providers in urban India: implications for interventions. J Int AIDS Soc. 2013;16(3 Suppl2):1-12.

4. Center, Miz Hasab. Perceived Stigmatization and Discrimination by Healthcare Providers towards Persons with HIV/AIDS. Addis Ababa: Intra Health International, USAIDS: Miz-Hasab Research Center; 2005.

5. Feyissa GT, Abebe L, Girma E, Woldie M. Stigma and discrimination against people living with HIV by healthcare providers, Southwest Ethiopia. BMC Public Health. 2012;12(1):522.

6. Zhiyong L. Legal Daily: HIV / AIDS patient get care difficulties highlights the lack of professional legislation. http://news.cntv.cn/china/20121123/101366. shtml. 2012.12.23.

7. Kemin Y. Why AIDS patients seek medical help difficulties? Discrimination eliminated need doctors to go first. http://jiankang.cntv.cn/2013/11/29/ ARTI1385687739410857.shtml. 2013.11.29.

8. Yan Ch. Kegiang Li dialogue anti-AIDS organizations: the whole society to jointly tackle the AIDS challenge http://news.cntv.cn/china/20121128/ 108094.shtml. 2012.11.28

9. Kamen C, Vorasarun C, Canning T, Kienitz E, Weiss C, Flores S, et al. The impact of stigma and social support on development of post-traumatic growth among persons living with HIV. J Clin Psychol Med Settings. 2016; 23(2):126-34

10. Wei W, Li XM, Harrison S, Zhao JF. The relationships between HIV stigma, emotional status, and emotional regulation among HIV affected children in rural China. AIDS Care. 2016;28(Suppl 2):161-7.

11. Audet CM, McGowan CC, Wallston KA, et al. Relationship between HIV stigma and self-isolation among people living with HIV in Tennessee. PLoS One. 2013;8(8):1.

12. Yanhai W, Ran H, Ran G, Arnade L. Discrimination against people with HIV/ AIDS in China. Equal Rights Rev. 2009;4:15-25.

13. Chinese legal information base. The National People's Congress of the People's Republic of China. http://law.npc.gov.cn:8081/FLFG/. Accessed 20 Dec 2017.

14. Yang Y, Zhang KL, Chan KY, Reidpath DD. Institutional and structural forms of HIV-related discrimination in health care: A study set in Beijing. AIDS Care. 2005;17(Supplement2):S129-40.

15. Peng $X$, Wanying $C$, Lahong J, et al. Evolution and perfection of the HIV /AIDS designated hospital system in China. Chinese J Health Policy. 2015; 8(5):67-72.

16. Banteyerga H, Kidanu A, Nyblade L, MacQuarrie K, Pande R. Yicha-laliko! Exploring HIV and AIDS stigma and related discrimination in Ethiopia: 
causes, manifestations, consequences, and coping mechanisms. Addis Ababa: MizHasab Research Center; 2004.

17. Hassan ZM, Wahsheh MA. Knowledge and attitudes of Jordanian nurses towards patients with HIV/AIDS: findings from a Nationwide survey. Issues in Ment Health Nurs. 2011;32(32):774-84.

18. Zarei N, Joulaei H, Darabi E, Fararouei M. Stigmatized attitude of healthcare providers: barrier for delivering health services to HIV positive patients. Int J Community Based Nurs Midwifery. 2015;3(4):292-300.

19. Stringer KL, Turan B, Mccormic LC, Durojaiye M. HIV-related stigma among healthcare providers in the deep south. AIDS Behav. 2015;20(1):115-25.

20. Bond V, Chase E, Aggleton P. Stigma, HIV/AIDS and prevention of mothertochild transmission in Zambia. Eval Progr Plann. 2002;25:347-56.

21. Earnshaw VA, Jin H, Wickersham J, Kamarulzaman A, John J, Altice FL, et al. Exploring intentions to discriminate against patients living with HIV/AIDS among future healthcare providers in Malaysia. Trop Med Int Health. 2014;19(6):672-9.

22. Harapan $\mathrm{H}$, Feramuhawan S, Kurniawan $\mathrm{H}$, Anwar S. HIV-related stigma and discrimination: a study of health care workers in Banda Aceh, Indonesia. Med J Indonesia. 2013;22(1):22.

23. Wagner AC, MCShane KE, Hart TA, Margolese S. A focus group qualitative study of HIV stigma in the Canadian healthcare system. Can J Hum Sex. 2016:25(1):61-71.

24. Andrewin A, Chien LY. Stigmatization of patients with HIV/AIDS among doctors and nurses in Belize. AIDS Patient Care STDs. 2008;22(11):897-906.

25. Mahendra VS, Gilborn L, Bharat S, Mudoi RJ, Gupta I, George B, et al. Understanding and measuring AIDS-related stigma in health care settings: a developing country perspective. SAHARA J. 2007;4(2):616-25.

26. Cai G, Moji K, Honda S, Wu X, Zhang K. Inequality and unwillingness to Care for People Living with HIV/AIDS: a survey of medical professionals in Southeast China. AIDS Patient Care STDs. 2007;21(8):593-601.

27. Kabbash IA, Abo Ali EA, Elgendy MM, Salem HM, Gouda MR, Elbasiony YS, et al. HIV/AIDS-related stigma and discrimination among health care workers at Tanta University hospitals, Egypt. Environ Sci Pollut Res. 2016;(5):1-8

28. Li L, Zunyou W, Sheng W, Zhaoc Y, Jia M, Yanet Z ea. HIV-related stigma in health care settings: a survey of service providers in China. AIDS Patient Care STDs. 2007;21(10):753-62.

Ready to submit your research? Choose BMC and benefit from:

- fast, convenient online submission

- thorough peer review by experienced researchers in your field

- rapid publication on acceptance

- support for research data, including large and complex data types

- gold Open Access which fosters wider collaboration and increased citations

- maximum visibility for your research: over $100 \mathrm{M}$ website views per year

At BMC, research is always in progress.

Learn more biomedcentral.com/submissions 